\title{
Scattering anisotropy measurements in dental tissues and biomaterials
}

\author{
A. Fernández-Oliveras \\ alilia@ugr.es
}

\section{Rubiño}

M. M. Perez

\author{
Departamento de Óptica, Facultad de Ciencias, Universidad de Granada, Campus Fuentenueva s/n. \\ 18071 Granada, Spain \\ Departamento de Óptica, Facultad de Ciencias, Universidad de Granada, Campus Fuentenueva s/n. \\ 18071 Granada, Spain \\ Departamento de Óptica, Facultad de Ciencias, Universidad de Granada, Campus Fuentenueva s/n. \\ 18071 Granada, Spain
}

Understanding the behaviour of light propagation in biological materials is essential for biomedical engineering and applications, and even more so when dealing with incoming biomaterials. Many methods for determining optical parameters from biological media assume that scattered light is isotropically distributed over all angles. However, an angular dependence of light scattering may exist and affect the optical behaviour of biological media. The present work seeks to experimentally analyze the scattering anisotropy in different dental tissues (enamel and dentine) and their potential substitute biomaterials (hybrid dental-resin, nano-filled composite, and zirconia ceramic) and comparatively study them. Coniometric measurements were made for four wavelengths in the visible range, allowing a spectral characterization of the materials studied. Previously, for each material, measurements were made with two different sample thicknesses at the same wavelength, checking the behaviour of the angular scattering profile. The asymmetry of experimental phase functions was considered in the recovery of the scattering anisotropy factor. The results demonstrate that the thicker sample yielded a less forwarddirected scattering profile than did the thinner sample. The biomaterials analysed show angular scattering comparable to those of the tissues that they may replace. Comparisons can be made by virtue of the low uncertainties found.

[DOI: http://dx.doi.org/10.2971/jeos.2012.12016]

Keywords: optical properties, scattering anisotropy, goniometric measurements, dental tissues, biomaterials

\section{INTRODUCTION}

Knowledge of the optical properties of biological structures is useful for clinical applications, and even more so when dealing with incoming biomaterials engineered to benefit the patient.

The optical properties of turbid media are described by the absorption coefficient, the scattering coefficient $\left(\mu_{s}\right)$, and the phase function, which represents the scattering angular distribution. The phase function is usually characterized by the scattering anisotropy factor $g$, which equals the average cosine of the scattering angle. For media with anisotropic scattering, gand the scattering coefficient can be combined into a reduced scattering coefficient, $\mu_{s}^{\prime}=(1-\mathrm{g}) \mu_{s}$, to effectively assume that the media exhibits isotropic scattering.

Certain methods for determining optical parameters assume that light scattered from biological media is isotropically distributed over all angles [1]-[3]. However, many works show that an angular dependence of light scattering may exist and affect the optical behaviour of biological media [4]-[11]. Therefore, the recovery of the scattering-angular distribution is important for biological media and, consequently, a better knowledge of the scattering anisotropy in biomaterials becomes of great interest.
In restorative dentistry, dental-resin composites are the most commonly used materials for replacing dental tissue, such as enamel. In recent years, dental nano-filled resin composites were introduced [12]. This dental-resin nanotechnology has provided fillers with considerably smaller particles, which can be dispersed in high concentrations and polymerized with molecules designed to be compatible when coupled with a polymer. As this molecular manufacturing provides other distinctive characteristics, optical properties of nanocomposites could differ from those of traditional composites, such as hybrids ones.

On the other hand, one ceramic material currently used to replace dentine in an irreversibly diseased tooth is yttrium cation-doped tetragonal zirconia polycrystal (3Y-TZP) because of its good mechanical properties [13]. However, its optical properties have not been fully studied.

The fulfilment of optimal quality and final success in medical application of biomaterials required complete studies to assess the appropriate material properties. In this sense, comparative analysis of optical behaviour in dental tissues and their substitute material becomes necessary.

The aim of this work is to experimentally analyse the scatter- 


\begin{tabular}{|c|c|c|c|c|}
\hline Dental-Resin Composite & Organic Matrix & Inorganic Filler & Filler Particle Size $(\mu \mathrm{m})$ & Type \\
\hline Filtek Supreme XT & $\begin{array}{c}\text { Bis-GMA, Bis-EMA, } \\
\text { UDMA, TEGDMA }\end{array}$ & $\begin{array}{c}\text { Silica agglomerate, } \\
\text { highly dispersed silica }\end{array}$ & $0.6-1.4$ & Nano-composite \\
\hline Tetric EvoCeram & $\begin{array}{c}\text { Bis-GMA, UDMA, } \\
\text { TEGDMA }\end{array}$ & $\begin{array}{c}\text { Ba glass, } \\
\text { ytterbium triflouride, } \\
\text { mixed oxides, } \\
\text { pre-polymers }\end{array}$ & Hybrid & $0.04-3$ \\
\end{tabular}

TABLE 1 Characteristics of the two different types of dental resin-composites analysed according to the manufacturer data

ing anisotropy in human enamel and dentine and their potential substitute biomaterials (hybrid dental-resin, nano-filled composite, and zirconia ceramic) and comparatively study them.

The scattering anisotropy factor can be experimentally determined by irradiating the media with a laser beam and making angular scattering measurements in a goniometer [4]-[7]. Since the scattering coefficient depends on the wavelength considered, in order to compute the reduced scattering coefficient, the corresponding $g$ value should be known. However, the spectral variation of the scattering anisotropy factor has not been fully analysed.

In the present work, goniometric measurements were made for four wavelengths in the visible range, allowing a spectral characterization of the material studied. Previously, for each material, measurements were made with two different sample thicknesses at the same wavelength, checking the behaviour of the angular scattering profile. The extrapolation of these scattering patterns to an incremental thickness would specify the appropriate phase function to be used in the radiative-transport equation [4]. With the intention of establishing comparisons between different materials, measurements were made with the most similar sample thicknesses for the rest of the wavelengths.

\section{THEORETICAL BACKGROUND}

When light travels through a biological media, it can be reflected, transmitted, or scattered due to the heterogeneity of the medium. When light finds an obstacle in the medium, a scattering event occurs, and the light-propagation direction changes; if light finds another obstacle, a new scattering event takes place and the light-propagation direction changes again. Scattering depends on the wavelength of irradiation, the refractive indexes of the medium, and the particle which causes the scattering, as well as on the particle diameter and cross section.

When light is scattered by a particle, its trajectory is deflected by an angle called scattering angle $(\theta)$. A value $\theta=0$ means that photons continue in the same direction as before the collision. The component of the new trajectory which is aligned in the forward direction is proportional to the scattering-angle cosine. The scattering anisotropy factor $g$ is a measure of the amount of forward direction retained after a single scattering event, and is defined by the mean value of the scattering-angle cosine:

$$
g=\int_{-1}^{1} p(\cos \theta) \cos \theta d(\cos \theta)
$$

where $p$ is the scattering-phase function that describes the fraction of light scattered from the obstacle as a function of the scattering angle. The phase function is normalized so that its integral over all directions is one:

$$
\int_{-1}^{1} p(\cos \theta) d(\cos \theta)=1
$$

Being normalized, $p(\theta)$ represents the probability of $\theta$ being the angle between the direction of the photons before and after the scattering event. The anisotropy factor varies between complete backward scattering $(g=-1)$ and complete forward scattering $(g=1)$. If $g=0$, then the medium is said to be isotropic, signifying that photons have the same probability of going in any direction.

The phase function describes the fraction of light scattered from one direction into another one and depends only on the angle between the two directions (scattering angle). Theoretically the scattering profile is independent of the incoming light direction. This means that the phase function should be symmetric when considering different directions of rotation with respect to the incident direction of light. Therefore, when goniometric measurements are made in order to determine the scattering profile, the direction of rotation is usually chosen arbitrarily.

In this work, for goniometric measurements, we have considered both directions of rotation in the same plane and proved that experimental phase functions are not symmetric. Taking this into account, to compute the $g$ factor that represents the phase function, we consider the average between the two values corresponding to both angular directions of rotation. Many works deal with scattering anisotropy in turbid media but do not in general consider the asymmetry of experimental phase functions in the recovery of the $g$ factor by goniometric measurements [5]-[7].

\section{MATERIALS AND METHODS}

\subsection{Sample preparation}

Two different types of dental-resin composites (nano-filled and hybrid) with similar polymeric matrixes (dimethacrylates): bisphenol A diglycidylether methacrylate (Bis-GMA), 


\begin{tabular}{|c|c|c|}
\hline Material & \multicolumn{2}{|c|}{ Thicknesses $(\mathrm{mm})$} \\
\hline Enamel & 0.97 & 0.46 \\
\hline Dentine & 0.88 & 0.41 \\
\hline Nanocomposite & 1.11 & 0.46 \\
\hline Hybrid composite & 1.03 & 0.46 \\
\hline Zirconia ceramic & 0.30 & 0.50 \\
\hline
\end{tabular}

TABLE 2 Characteristics of the two different types of dental resin-composites analysed according to the manufacturer data

bisphenol A polyethylene glycol diether dimethacrylate (BisEMA), urethane dimethacrylate (UDMA) and triethylene glycol dimethacrylate (TEGDMA), were studied. The characteristics of each dental-resin composite, according to the manufacturer, are shown in Table 1.

Specimens were made on a glass plate (Knittel GLASER, Bielefeld, Germany) with a circular hole prepared with a highspeed hand-piece and a round bur. With the use of glass plates $1 \mathrm{~mm}$ and $0.5 \mathrm{~mm}$ thick, two samples of different thickness were prepared from each dental resin. After the placement of composites, a clear plastic sheet (Acrylite Plus Clear, Tap Plastics, Dublin, CA, USA) was laid on the top and bottom of the mould and another glass plate was pressed onto the top to standardize the specimen thickness. Each sample underwent photo-polymerization for 40 seconds using a light-curing unit (Bluephase, Ivoclar Vivadent AG, Liechtenstein) with a irradiance of $1100 \mathrm{~mW} / \mathrm{cm}^{2} \pm 10 \%$. After photo-polymerization, all glass plates were removed. Specimens were handled according to manufacturer's instructions. All specimens were prepared by the same user in order to minimize variability.

For dental-tissue sample preparation, one human tooth was laterally cut into slices. All cuts were made with an automatic precision cut-off machine (Accutom-5 Struers, Ballerup, Denmark). The slices provided samples of enamel and dentine with different thicknesses. Specimens were polished with silicon carbide paper from 220 to 4000 grits and finally with alumina slurry of $1,0.3$ and $0.05 \mu \mathrm{m}$. Then, they were placed in an ultrasonic cleaner (Renfert, CA, USA) with distilled water in 3 cycles of $10 \mathrm{~min}$ to eliminate polishing detritus. Finally, the samples were stored in distilled water.

Sintered LAVA ${ }^{T M}$ Zirconia samples provided by the manufacturer with two different thicknesses were used to study zirconia ceramic material. The framework ceramics were fabricated using computer-aided design and manufacturing (CAD/CAM) procedures from presintered zirconia blanks, the sizes of which had been increased to compensate for shrinkage during sintering in a special high-temperature furnace.

\subsection{Experimental setup}

The set-ups used for scattering anisotropy measurements schematically (Figures 1 and 2) consisted of a randomly polarized laser source, a goniometric rotary stage of $120 \mathrm{~mm}$ in diameter (NT62-295, Edmund Optics, USA) and a photodiode detector connected to an amplifier-multimeter (34401A, Agilent Technologies, USA) measuring system. The photodiode

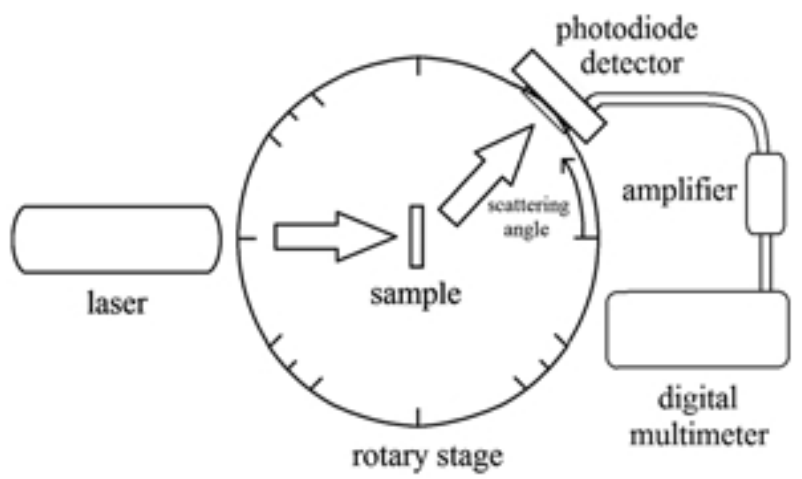

FIC. 1 Experimental set-up used in scattering anisotropy measurements, for scattering angles between 0 and $156^{\circ}$, and between 0 and $-162^{\circ}$.

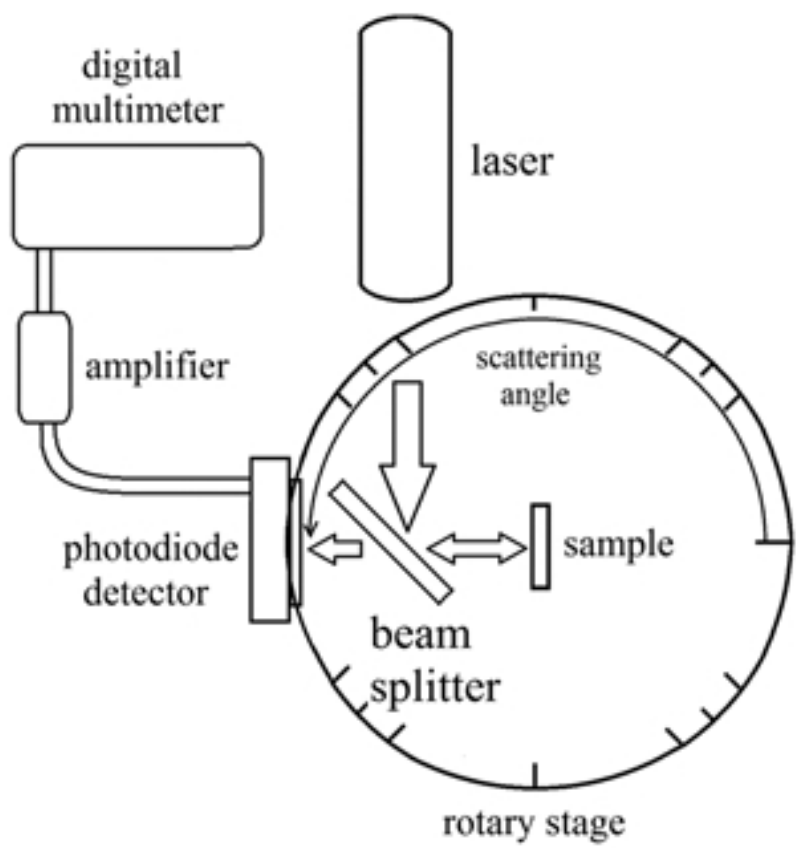

FIG. 2 Experimental set-up used in scattering anisotropy measurements, for scattering angles between 160 and $180^{\circ}$, and between -164 and $-180^{\circ}$.

was mounted at the edge of the rotary stage and had an aperture of $3 \mathrm{~mm}$ in diameter. The samples were placed in the centre of the rotary stage and irradiated with the laser beam. The laser light scattered off the sample was detected by the photodiode for different scattering angles. With each sample, measurements were made in both directions of rotation (between 0 and 180 degrees, and between 0 and -180 degrees).

In the case of the backward-forward scattering measurements, with the set-up configuration shown in Figure 1, the detector itself may prevent the laser beam from reaching the sample. To avoid this drawback, we propose another configuration where a beam splitter allows the laser beam to aim at the sample by reflection without blocking backscattered light, which reached the detector by transmission (Figure 2). This set-up configuration was used for the measurements at scattering angles between 160 and $180^{\circ}$, and between -164 and $-180^{\circ}$.

The scattering anisotropy factor was calculated for the specimens at different wavelengths. For this, measurements were made using two laser sources: a He-Ne laser source with a power of $17.0 \mathrm{~mW}$ and beam diameter of $0.98 \mathrm{~mm}$ (LHRR- 
1700, Research Electro Optics, USA), which provided a wavelength of $632.8 \mathrm{~nm}$; and a tunable ion-argon laser source with $1000 \mathrm{~mW}$ of maximum total power and beam diameter of 0.75 mm (Stellar-Pro-L ML/1000, Modu-Laser, USA), which provided wavelengths of $457.9,488.0$ and $514.5 \mathrm{~nm}$.

First, with the He-Ne laser, measurements were made for each material with two different sample thicknesses (shown in Table 2) in order to check the behaviour of the angular scattering profile. Then, with the ion-argon laser, measurements were made with the most similar sample thickness (third column in Table 2), to establish comparisons between different materials.

\subsection{Data processing}

The data of detected values represented the collected power of the scattering signal. From the measurements of scatteredlight signal $(V)$ at various scattering angles $(\theta)$, the scattering anisotropy factor $(g)$ was calculated according to [6]:

$$
g=\frac{\sum_{i} V_{i} \cos \theta_{i}}{\sum_{i} V_{i}}
$$

where the sums were taken over the evaluated scattering angles and signal values, at an increment of 4 degrees of scattering angle.

Measurements were made in both directions of rotation (between 0 and 180 degrees, and between 0 and -180 degrees). Considering positive and negative angles, we calculated both corresponding $g$ values and determined the average $g$. The average deflection angle in a scattering event (average scattering angle) can be calculated as the arccosine of $g$, since $g$ represents the average cosine of the scattering angle. Therefore, from the average $g$ value, the related average scattering angle was determined. The uncertainties associated to the anisotropy-factor values and the average scattering angles were calculated taking into account the law of propagation of uncertainties [14].

For the scattering angles between 160 and $180^{\circ}$, and between -164 and $-180^{\circ}$, measurements were made under different experimental conditions since we used another set-up (shown in Figure 2). The scattered light signals were normalized in order to add the measurements to the rest of the data (collected with the set-up in Figure 1). The normalizing factor was estimated in this way: for three different scattering angles, measurements of the scattering signal were made with both experimental set-ups. For each pair of measurements, a value of the normalizing factor was calculated and the average was finally considered. Each normalizing factor value was determined by dividing the scattering signal measured with the set-up in Figure 1 by the scattering signal measured with the set-up in Figure 2 . The uncertainty in the normalizing factor was also calculated according to the law of propagation of uncertainties and considered in the computation of the uncertainties associated with the normalized scattering signal values (those corresponding to scattering angles between 160 and $180^{\circ}$, and between -164 and $-180^{\circ}$ ).
As a test of reproducibility, for the zirconia ceramic sample at $488.0 \mathrm{~nm}$, measurements with the set-up proposed (Figure 2) were repeated, demonstrating that the difference between the corresponding $g$ values was less than the associated uncertainty.

To investigate the significance of the signal values measured with the set-up proposed for angles between 160 and $180^{\circ}$, and between -164 and $-180^{\circ}$, we also computed the $g$ values while omitting these measurements. The difference between the $g$ values determined (with and without these measurements) was found to be significant, for each material studied.

Before processing, the data were corrected to reduce effects from the background noise, due to laboratory ambient lighting and photodiode dark current (response produced in the absence of input light-signal, i.e. with the detector in the dark). The background noise was corrected by subtracting from the measured signal values the background signal detected when obscuring the laser source.

\section{RESULTS AND DISCUSSION}

To make comparisons between the scattering-signal profiles found at different wavelengths and with different sample thicknesses, we normalized the scattering-signal profiles. For positive scattering angles, the profile was normalized by dividing the each signal value by the sum of all the signal values from 0 to 180. For negative scattering angles, the profile was normalized by dividing each signal value by the sum of all the signal values from 0 to -180 . In this way, we determined the normalized phase function from the angular scattering profile. Each value of the normalized phase function represents the probability associated to the corresponding scattering angle as the deflection angle in a scattering event.

\subsection{Influence of sample thickness}

As pointed out above, to check the influence of the sample thickness in the angular scattering profile, with the He-Ne laser, we made measurements for each material with two different sample thicknesses (shown in Table 2).

The scattering phase functions found with the different sample thicknesses of each material are shown in Figures 3 to 7. The corresponding average $g$ values and the related average scattering angles are listed in Table 3, with their associated uncertainties. It is noteworthy that by virtue of the low uncertainties found, differences between the $g$ values are significant.

The results show that, for all specimens studied, the thicker the sample the lower the $g$ value obtained from goniometric measurements, in accordance with conclusions of other works [4]. As established, the thicker sample attenuates the on-axis intensity signal, yielding a less forward-directed scattering profile than the thinner sample. The extrapolation of these scattering profiles to an incremental thickness would specify the appropriate phase function to be used in the radiativetransport equation. 


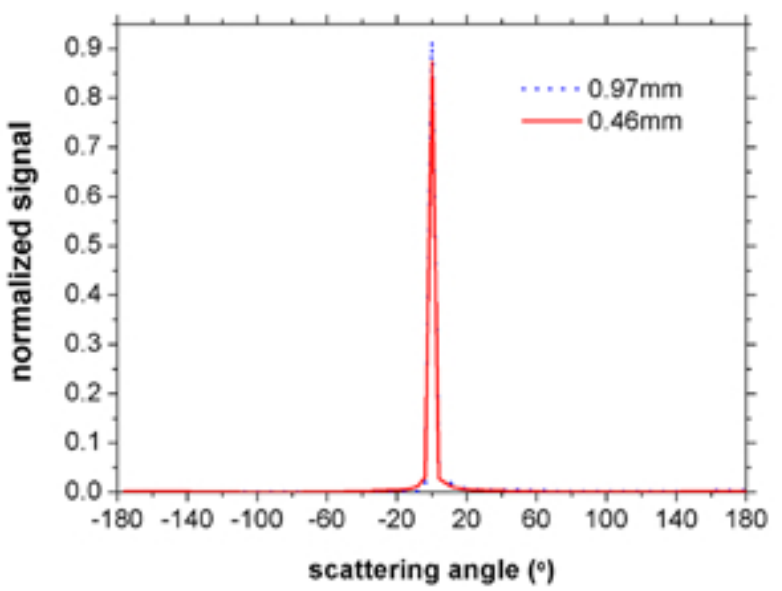

FIG. 3 Normalized scattering signal for the human enamel samples of different thicknesses.

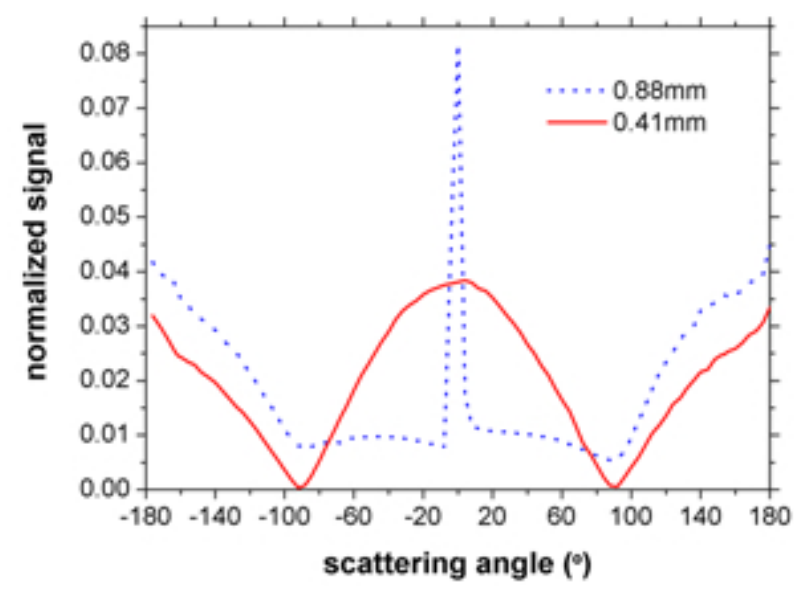

FIG. 4 Normalized scattering signal for the human dentine samples of different thicknesses.

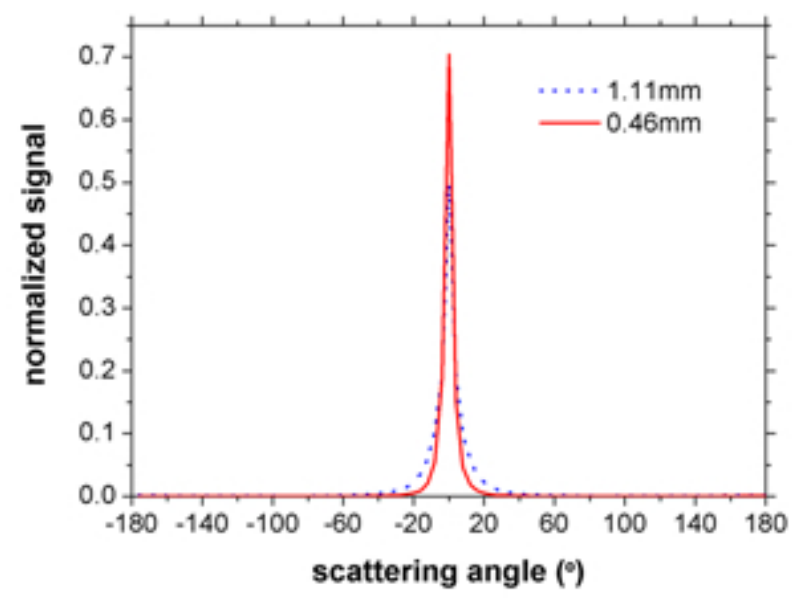

FIC. 5 Normalized scattering signal for the dental nanocomposite samples of different thicknesses.

Therefore, in the subsequent measurements, we used the thinner sample of each material, except in the case of the zirconia ceramic; for zirconia ceramic, since differences between the two sample thicknesses were lower, we chose the thicker one because its thickness was more similar to those of the thinner samples of the other materials.

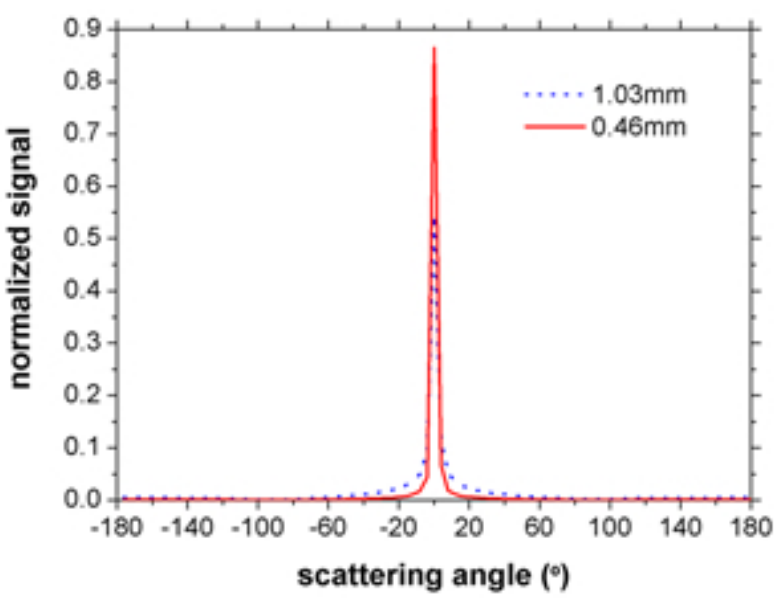

FIG. 6 Normalized scattering signal for the hybrid composite samples of different thicknesses.

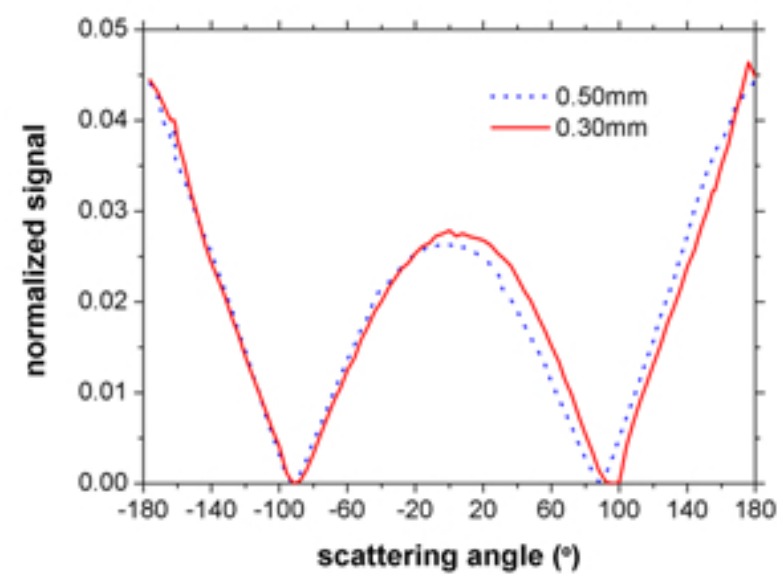

FIC. 7 Normalized scattering signal for the zirconia ceramic samples of different thicknesses.

\subsection{Spectral variation}

In Figures 8 to 12, for each material, the scattering phase functions at the evaluated wavelength are shown. The average $g$ values calculated from the measurements and the related average scattering angles with their uncertainties associated are listed in Tables 4 and 5. It is worth noting the low uncertainty found, which allowed the $g$ values calculated to be distinguished and compared.

The results reveal that $g$ values have similar spectral variation for all the material, except for the human dentine. For the dental-resin composites, human enamel, and zirconia ceramic, the $\mathrm{g}$ values diminish at $488.0 \mathrm{~nm}$ and augment from 514.5 $\mathrm{nm}$. For the human dentine, the $\mathrm{g}$ value increase at $488.0 \mathrm{~nm}$ and decrease from $514.5 \mathrm{~nm}$. This may be due to the different internal structure of dentine, which is composed of the socalled tubules, which are oriented from the pulp to the dentinenamel junction [15].

Instead, dental enamel is internally composed of inorganic apatite-like crystals surrounded by a protein-lipid-water matrix, with the crystals clustered together in rods approximately perpendicular to the tooth surface [16]. Compared with the dentine structure, the enamel structure is more similar to the 


\begin{tabular}{|c|c|c|c|c|c|c|}
\hline Material & Thickness $(\mathrm{mm})$ & Average $g$ & Average $\theta\left(^{\circ}\right)$ & Thickness $(\mathrm{mm})$ & Average $g$ & Average $\theta\left(^{\circ}\right)$ \\
\hline Enamel & 0.97 & $0.8870 \pm 0.0003$ & $27.51 \pm 0.04$ & 0.46 & $0.91867 \pm 0.00011$ & $23.268 \pm 0.016$ \\
\hline Dentine & 0.88 & $-0.3021 \pm 0.0020$ & $107.59 \pm 0.12$ & 0.41 & $0.0620 \pm 0.0010$ & $86.45 \pm 0.06$ \\
\hline $\begin{array}{c}\text { Nano- } \\
\text { composite }\end{array}$ & 1.11 & $0.9283 \pm 0.0004$ & $21.83 \pm 0.06$ & 0.46 & $0.98297 \pm 0.00021$ & $10.59 \pm 0.07$ \\
\hline $\begin{array}{c}\text { Hybrid } \\
\text { composite }\end{array}$ & 1.03 & $0.7813 \pm 0.0004$ & $38.62 \pm 0.04$ & 0.46 & $0.95429 \pm 0.00014$ & $17.39 \pm 0.03$ \\
\hline $\begin{array}{c}\text { Zirconia } \\
\text { ceramic }\end{array}$ & 0.30 & $-0.1763 \pm 0.0014$ & $100.15 \pm 0.08$ & 0.50 & $-0.2074 \pm 0.0024$ & $101.97 \pm 0.14$ \\
\hline
\end{tabular}

TABLE 3 Average $g$ values and scattering angles with their associated uncertainties for the different sample thicknesses $(\lambda=632.8 \mathrm{~nm})$.

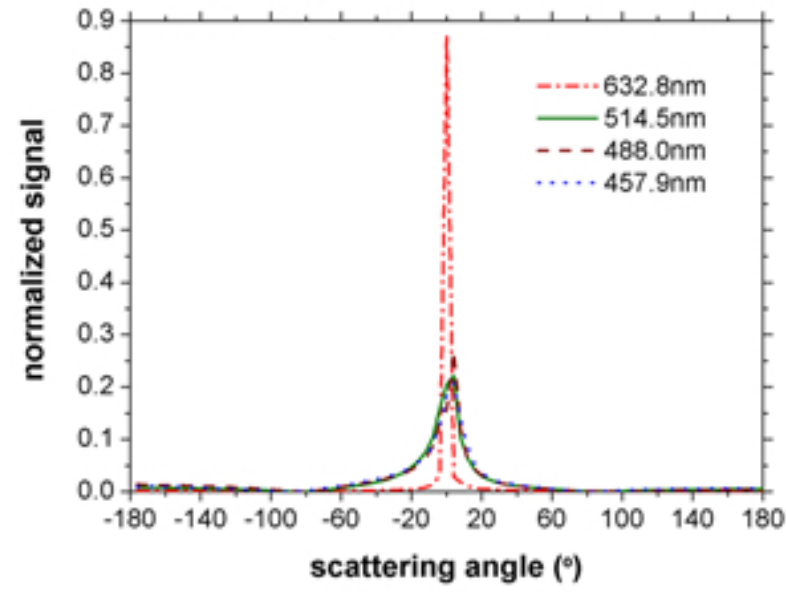

FIG. 8 Normalized scattering signal of the human enamel at different wavelengths of the visible spectrum.

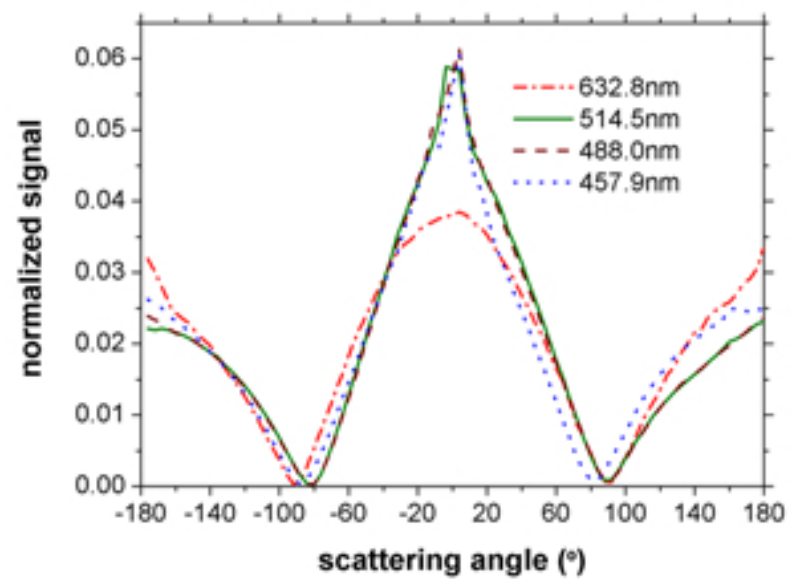

FIG. 9 Normalized scattering signal of the human dentine at different wavelengths of the visible spectrum.

dental-resin composite structure (since dental-resins are composed by inorganic filler particles immersed in an organic matrix), and also to the microstructure of 3Y-TZP ceramics for dental applications, which consists of small equiaxed grains with diameter sizes depending on the sintering temperature [17].

For the dental composites, the angular scattering profiles indicate the presence of a more pronounced forward-directed scattering in the nano-filled dental resin than in the hybrid dentalresin composite. The results show that $g$ values are higher for the nanocomposite than for the hybrid composite, at every

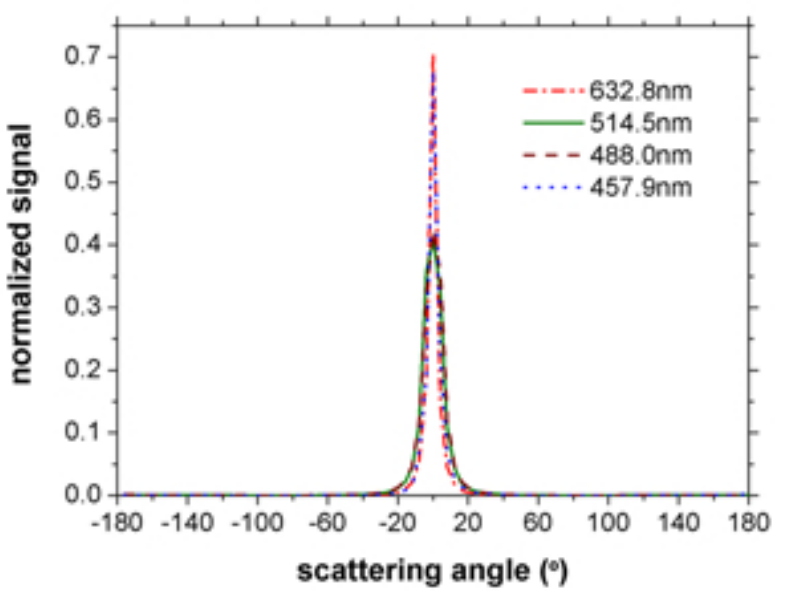

FIG. 10 Normalized scattering signal of the dental nanocomposite at different wavelengths of the visible spectrum.

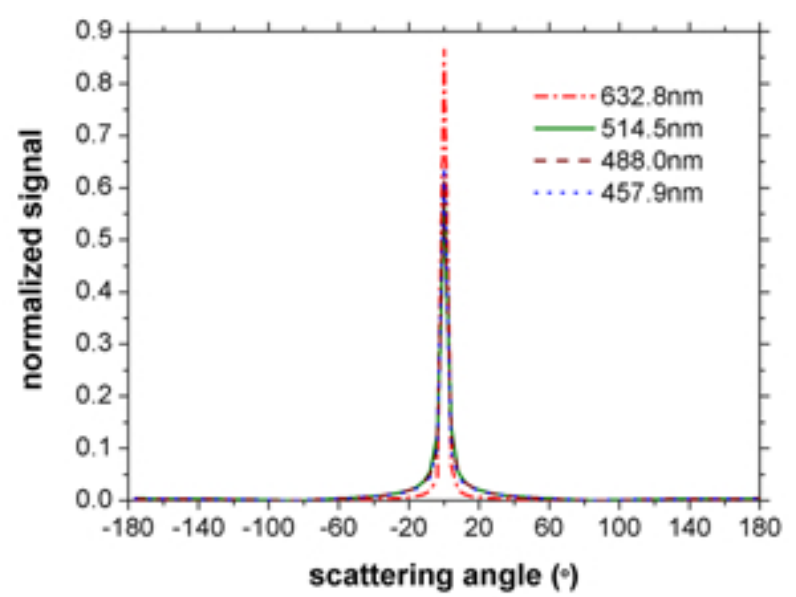

FIG. 11 Normalized scattering signal of the hybrid composite at different wavelengths of the visible spectrum.

wavelength studied. The higher $g$ values for the nanocomposite reveal that the scattering particle is more similar to a perfect sphere, since it is established that for more spherical scattering particles the scattering anisotropy factor increases [5]. On the other hand, for spherical nanoparticles (diameters from 0.05 to $2.5 \mu \mathrm{m}$ ) in aqueous suspensions, it is established that the $g$ value increases with the scatterer diameter [18]. Therefore, lower $g$ values for the hybrid composite may be related to a lower concentration of scattering centres, resulting in a smaller scatterer diameter. This agrees with observations made elsewhere, when filler particle size and particle-size dis- 


\begin{tabular}{|c|c|c|c|c|c|}
\hline \multirow{2}{*}{$\lambda(\mathrm{nm})$} & \multicolumn{5}{|c|}{ Average $g$} \\
\cline { 2 - 6 } & Enamel & Dentine & Nanocomposite & Hybrid composite & Zirconia ceramic \\
\hline 457.9 & $0.6812 \pm 0.0008$ & $0.1291 \pm 0.0010$ & $0.9617 \pm 0.0003$ & $0.8710 \pm 0.0003$ & $-0.192 \pm 0.003$ \\
\hline 488.0 & $0.6666 \pm 0.0006$ & $0.2104 \pm 0.0009$ & $0.9487 \pm 0.0004$ & $0.8555 \pm 0.0003$ & $-0.247 \pm 0.005$ \\
\hline 514.5 & $0.7418 \pm 0.0006$ & $0.2122 \pm 0.0010$ & $0.9570 \pm 0.0004$ & $0.8558 \pm 0.0003$ & $-0.233 \pm 0.003$ \\
\hline 632.8 & $0.91867 \pm 0.00011$ & $0.0620 \pm 0.0010$ & $0.98297 \pm 0.00021$ & $0.95429 \pm 0.00014$ & $-0.2074 \pm 0.0024$ \\
\hline
\end{tabular}

TABLE 4 Average $g$ values with their associated uncertainties for the different wavelengths analysed.

\begin{tabular}{|c|c|c|c|c|c|}
\hline \multirow{2}{*}{$\lambda(\mathrm{nm})$} & \multicolumn{5}{|c|}{ Average $\theta\left(^{\circ}\right)$} \\
\cline { 2 - 6 } & Enamel & Dentine & Nanocomposite & Hybrid composite & Zirconia ceramic \\
\hline 457.9 & $47.06 \pm 0.06$ & $82.58 \pm 0.06$ & $15.91 \pm 0.06$ & $29.42 \pm 0.03$ & $101.08 \pm 0.17$ \\
\hline 488.0 & $48.19 \pm 0.05$ & $77.86 \pm 0.05$ & $18.44 \pm 0.07$ & $31.19 \pm 0.03$ & $104.3 \pm 0.3$ \\
\hline 514.5 & $42.11 \pm 0.05$ & $77.75 \pm 0.06$ & $16.87 \pm 0.08$ & $31.16 \pm 0.04$ & $103.48 \pm 0.20$ \\
\hline 632.8 & $23.268 \pm 0.016$ & $86.45 \pm 0.06$ & $10.59 \pm 0.07$ & $17.39 \pm 0.03$ & $101.97 \pm 0.14$ \\
\hline
\end{tabular}

TABLE 5 Average scattering angles with their associated uncertainties for the different wavelengths analysed.

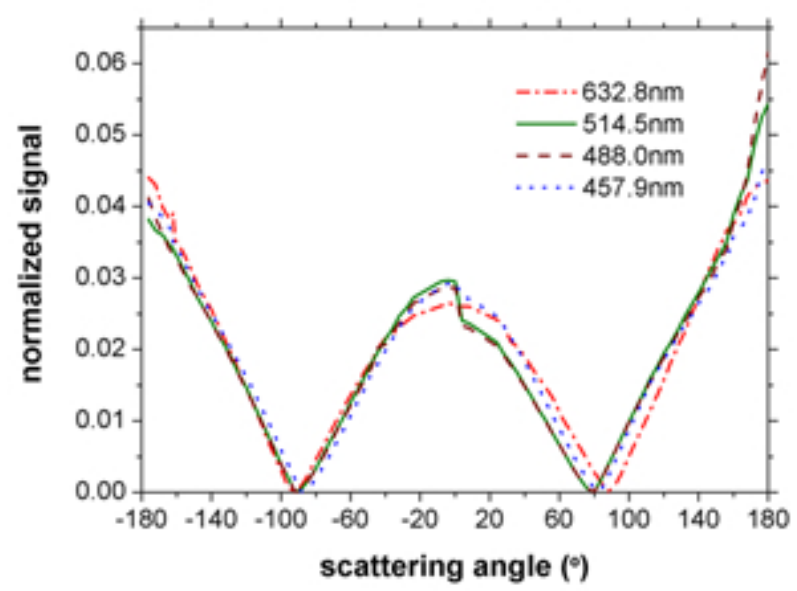

FIG. 12 Normalized scattering signal of the zirconia ceramic at different wavelengths of the visible spectrum.

tribution of dental-resin composites was determined employing a digital-image analysis [19].

From the scattering profiles of Figures 8 to 12 and the $g$ values of Table 5, we can conclude that, in general terms, the dentalresin composites and human enamel show a similar angular scattering behaviour; moreover, on the other hand, the zirconia ceramic present a scattering angular behaviour more similar to that of human dentine. This is valuable for biomedical applications, since it means that the biomaterials analysed show angular-scattering behaviour comparable to that of the tissues that they are meant to replace.

\section{CONCLUSIONS}

The phase function that represents the scattering angular distribution is usually characterized by the anisotropy factor $g$, which equals the average cosine of the scattering angle. The value of $g$ can be determined experimentally by irradiating the material with a laser beam and making angular scattering measurements in a goniometer.

We have experimentally analysed the scattering anisotropy in human enamel and dentine and their potential substitute biomaterials (hybrid dental-resin, nano-filled composite, and zirconia ceramic) and comparatively study them.

For goniometric measurements, we considered both directions of rotation in the same plane and proved that experimental phase functions are not symmetric. Taking this into account, to compute the $g$ factor that represents the phase function, we considered the average between the two values corresponding to both angular directions of rotation.

In the case of the backward-forward scattering measurements, the detector itself may prevent the laser beam from reaching the sample. To avoid this drawback, we propose another configuration where a beam splitter allows the laser beam to aim at the sample by reflection without blocking backscattered light, which reaches the detector by transmission. This setup was used for the measurements at scattering angles of between 160 and $180^{\circ}$, and between -164 and $-180^{\circ}$. When these measurements were omitted, the $g$ values were also computed and the differences were found to be significant for each material studied.

Since the scattering coefficient depends on the wavelength, in order to compute the reduced scattering coefficient, the corresponding $g$ value should be known. In this work, angularscattering measurements were made for four wavelengths in the visible range, allowing a spectral characterization of the material studied. Previously, for each material, measurements were made with two different sample thicknesses at the same wavelength, checking the behaviour of the angular-scattering profile.

The results demonstrated that the thicker sample yielded a less forward-directed scattering profile than did the thinner sample. The scattering anisotropy factor showed similar spectral variation for all the material, except for the human dentine. The differences found for human dentine may be due to the different internal structure of dentine, which is an anisotropic tissue compared with the other materials investigated. Human dentine consists of closely packed dentin tubules traversing all the way from the pulp to the enamel 
in all directions. Having different slices from the same tooth could result in different structures. This fact may have influence on the results found for human dentine. Comparison can be made by virtue of the low uncertainties associated to the $g$ values found.

It is worth noting that, in general terms, the dental-resin composites and the human enamel showed a similar angular scattering behaviour; on the other hand, the zirconia ceramic presented a scattering angular behaviour more similar to those of the human dentine. This is valuable for biomedical applications, since it means that, in terms of angular scattering behaviour, the biomaterials are comparable to the tissues that they are meant to replace.

\section{ACKNOWLEDGEMENTS}

This research is supported by Grant MAT2009-09795 from the Spanish Ministry of Science and Innovation.

A. Fernández-Oliveras is recipient of a FPU PhD Scholarship from the Spanish Ministry of Science and Innovation.

The authors would like to thank Dr. Scott A. Prahl for stimulating discussions and helpful suggestions on this work.

\section{References}

[1] R. R. Anderson, and J. J. Parish, "The optics of human skin," J. Invest. Dermatol. 77, 13-19 (1981).

[2] M. J. C. van Cemert, R. Verdaasdonk, E. G. Stassen, G. A. C. M. Schets, G. H. M. Gijsbers, and J. J. Bonnier, "Optical properties of human blood vessel wall and plaque," Laser. Surg. Med. 5, 235-237 (1985).

[3] S. Ertefai, and A. E. Profio, "Spectral transmittance and contrast in breast diaphanography," Med. Phys. 12, 393-400 (1985).

[4] S. L. Jacques, C. A. Alter, and S. A. Prahl, "Angular Dependence of HeNe Laser Light Scattering by Human Dermis," Lasers Life Sci. 1, 309-334 (1987).

[5] J. M. Steinke, and A. P. Shepherd, "Comparison of Mie theory and the light scattering of red blood cells," Appl. Optics 27, 4027-4033 (1988).

[6] D. K. Sardar, F. S. Salinas, J. J. Perez, and A. T. C. Tsin, "Optical characterization of melanin," J. Biomed. Opt. 6, 404-411 (2001).
[7] D. K. Sardar, R. M. Yow, A. T. C. Tsin, and R. Sardar, “Optical scattering, absorption and polarization of healthy and neovascularized human retinal tissues," J. Biomed. 0pt. 10, 0515011-1-8 (2005).

[8] N. Joshi, C. Donner, and H. W. Jensen, "Noninvasive measurement of scattering anisotropyin turbid materials by nonnormal incident illumination," Opt. Lett. 31, 936-938 (2006).

[9] M. Hammer, D. Schweitzer, E. Thamm, and A. Kolb, “Optical properties of ocular fundus tissues determined by optical coherence tomography," Opt. Commun. 186 149-153 (2000).

[10] A. Kienle, M. S. Patterson, L. Ott, and R. Steiner, "Determination of the scattering coefficient and the anisotropy factor from laser Doppler spectra of liquids including blood," Appl. Optics 35 , 3404-3412 (1996).

[11] I. Turcu, C. V. L. Pop, and S. Neamtu, "High-resolution angleresolved measurements of light scattered at small angles by red blood cells in suspension," Appl. Optics 45, 1964-1971 (2006).

[12] S. B. Mitra, D. Wu, and B. N. Holmes, "An application of nanotechnology in advanced dental materials," J. Am. Dent. Assoc. 134, 1382-1390 (2003).

[13] J. Chevalier, and L. Gremillard, "Ceramics for medical applications: A picture for the next 20 years," J. Eur. Ceram. Soc. 29, 1245-1255 (2009).

[14] International Organization for Standardization, Guide to the Expression of Uncertainty in Measurement (ISO, Geneva, 1993).

[15] I. A. Mjor, "The morphology of dentin and dentinogenensis" in Dentin and Dentino Genesis, A. Linde, ed., 2-18 (CRC Press, Boca Raton, 1984).

[16] M. E. J. Curzon, and J. D. B. Featherstone, "Chemical composition of enamel" in Handbook of Experimental Aspects of Oral Biochemistry, E. P. Lazzan, ed., 123-135 (CRC Press, Boca Raton, 1983).

[17] I. Denrya, and J. R. Kellyb "State of the art of zirconia for dental applications," Dent. Mater. 24, 299 (2008).

[18] J. D. Chicea, "Probing particle aggregation in aqueous suspensions by light scattering anisotropy measurements," Optoelectron. Adv. Mat. 12, 152 (2010).

[19] R. Ghinea, L. Ugarte-Alvan, A. M. Ionescu, J. C. Cardona, M. M. Pérez, and A. Yebra, "Influence of the size and distribution of filler particles on the colour of resin composites," in Proceeding of the Fourth European Conference on Color in Graphics, Imaging, and Vision, 296-299 (Society for Imaging Science and Technology, Terrasa, 2008). 